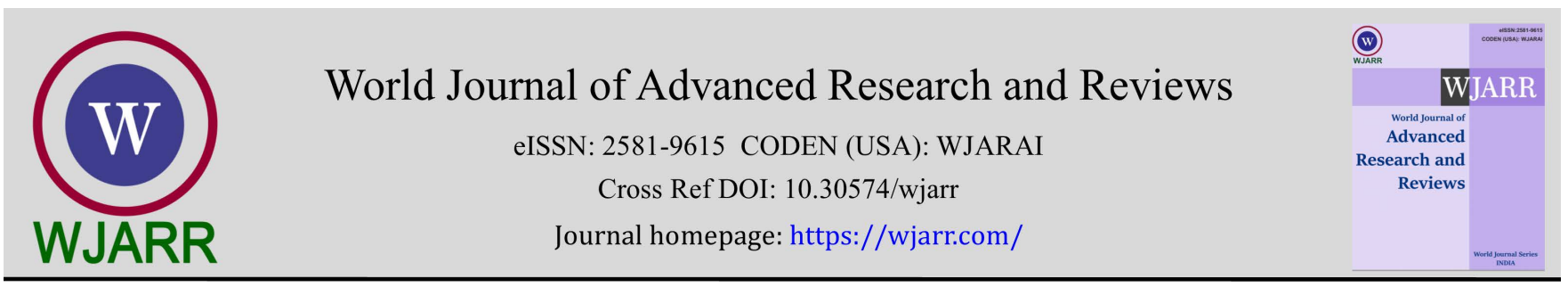

(CASE REPORT)

Check for updates

\title{
A child having rare Hereditary Multiple Exostosis with Covid-19 disease: A case study
}

\author{
Rummana Tazia Tonny ${ }^{1,}{ }^{*}$, Rifat Taher Anne ${ }^{1}$, Kazi Lutfar Rahman ${ }^{2}$ and Ferdousi Hasnat ${ }^{1}$ \\ ${ }^{1}$ Department of Pediatrics, Kurmitola General Hospital, Cantonment, Dhaka, Bangladesh. \\ ${ }^{2}$ RT- PCR lab, Kurmitola General Hospital, Cantonment, Dhaka, Bangladesh.
}

World Journal of Advanced Research and Reviews, 2022, 13(02), 320-324

Publication history: Received on 05 January 2022; revised on 12 February 2022; accepted on 14 February 2022

Article DOI: https://doi.org/10.30574/wjarr.2022.13.2.0138

\begin{abstract}
Hereditary Multiple Exostosis is a rare bone disease in pediatric age group. It is an autosomal dominant disorder, characterized by excessive bony overgrowth arising from lateral aspect of metaphysis of long bone, covered by a cartilaginous cap. These lesions remain asymptomatic clinically. When symptomatic, a variety of health hazards can arise like chronic pain, skeletal deformity, neuro-vascular compression etc. Malignant transformation is a rare, but feared complication. Diagnosis can be made by simple radiographic examination, when symptoms arise. Treatment is only conservative or surgical. SARS- CoV-2 may infect children with any pre-existing disease, like HME in the same proportion of healthy children. We report a rare case of hereditary multiple exostosis, simultaneously suffering from covid-19 disease, that has not been reported previously.
\end{abstract}

Keywords: Hereditary Multiple Exostosis (HME); Osteochondroma; SARS-CoV-2; Covid-19

\section{Introduction}

Hereditary Multiple Exostosis (HME), also known as familial osteochondromatosis, is a rare congenital pediatric disorder characterized by formation of multiple osteochondromas near the growth plate of long bones [2]. The condition is inherited as an autosomal dominant manner. The pathogenesis is linked to loss of functional mutation of 2 genes - Exostosin 1 (EXT1) and Exostosin -2 (EXT2) which are responsible for synthesis of heparan sulfate chain. The disease affects 1 in 50,000 births without any sexual predominance [1,3]. WHO defined osteochondroma as a cartilage - capped bony overgrowth that is benign in nature. It has 2 variants - sessile and pedunculated variety. The bony overgrowth is composed of cortex and a marrow cavity that is continuous with the host bone. The pedunculated variety is directed away from the growth plate and joint. The commonest location of exostoses is at the lateral side of the active growth plate of long bone. The number and size of osteochondromas increase during childhood and stops growing at puberty until closure of the growth plate [3]. Clinically most patients remain asymptomatic [6,7]. While symptomatic, it can cause a variety of health problems like skeletal deformity, chronic pain and functional impairment[1] . A rare, but important complication is malignant transformation during adulthood $[8,9]$.

We report a case of a 9 year old boy, admitted in a covid-19 dedicated hospital for being SARS-CoV-2 positive with mild symptoms, who was diagnosed first as a case of HME incidentally following trauma at the age of 3 years.

\section{Case Summary}

A 9-years-old boy of non- consanguineous parents who is admitted in a covid-19 dedicated hospital with the complaints of high grade, intermittent fever and non - productive cough without having any diurnal variation - for 3 days. He had

\footnotetext{
* Corresponding author: Rummana Tazia Tonny

Department of Pediatrics, Kurmitola General Hospital, Cantonment, Dhaka, Bangladesh.

Copyright $(2022$ Author(s) retain the copyright of this article. This article is published under the terms of the Creative Commons Attribution Liscense 4.0.
} 
history of close contact with covid positive patient as his father was also covid positive concomitantly. The boy had no history of runny nose, respiratory distress, chest pain, any contact with TB patient or any other problems.

Mother also complained of a hard, globular swelling of right middle phalanx of middle finger, occasionally causing pain. First swelling was identified over the right wrist joint following trauma at 3 years of his age. The size and number of swelling are increasing gradually, causing functional impairments such as walking difficulties, difficulties in sports and games. As a result, the boy adopted a sedentary life style, which was more pronounced during this pandemic situation and developed excessive weight gain. Moreover, his calorie intake was more than normal.

He had no previous history of fracture and no family history of bone disorder or a similar case in his family.

\section{Clinical exam}

Clinical examination found that the boy was obese, weight $52 \mathrm{~kg}$ (lies far above $97^{\text {th }}$ centile), height $136 \mathrm{~cm}$ (50 BMI $28.11 \mathrm{~kg} / \mathrm{m} 2$ (far above $97^{\text {th }}$ centile). Vital signs - temperature $101 \circ \mathrm{F}$, heart rate $110 \mathrm{~b} / \mathrm{min}, \mathrm{BP} 110 / 80 \mathrm{~mm}-\mathrm{Hg}$ (SBP lies in between $50^{\text {th }}-90^{\text {th }}$ centile, DBP on $95^{\text {th }}$ centile), respiratory rate $24 \mathrm{br} / \mathrm{min}$, SP02 $95 \%$ in room air, on chest auscultation-few creps were present initially during the hospital course. Locomotor system examination revealed a globular swelling over the middle phalanx of right middle finger (Figure 1B), hard and fixed to the deep plane, tender on gentle pressure. Another swelling of similar characteristics, but smaller size was also identified over the distal phalanx of $4^{\text {th }}$ digit of same side (Figure 1C). No other visible swelling present. Tenderness present over the different parts of the body including both shoulder joints, wrist joints, ribs near the costochondral junction. Knock knee present (Figure 1D), having no leg-length discrepancy. Other system examinations reveal normal findings.
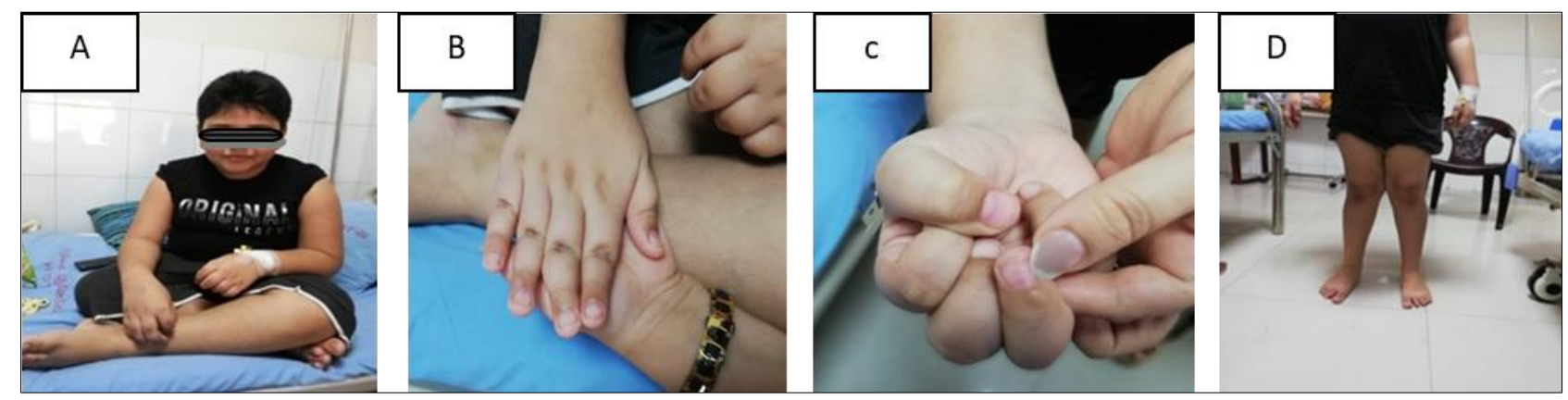

Figure 1 Bony swelling located on middle phalanx of right middle finger (B), distal phalanx of right $4^{\text {th }}$ digit (C) and genu valgum deformity is shown (D)
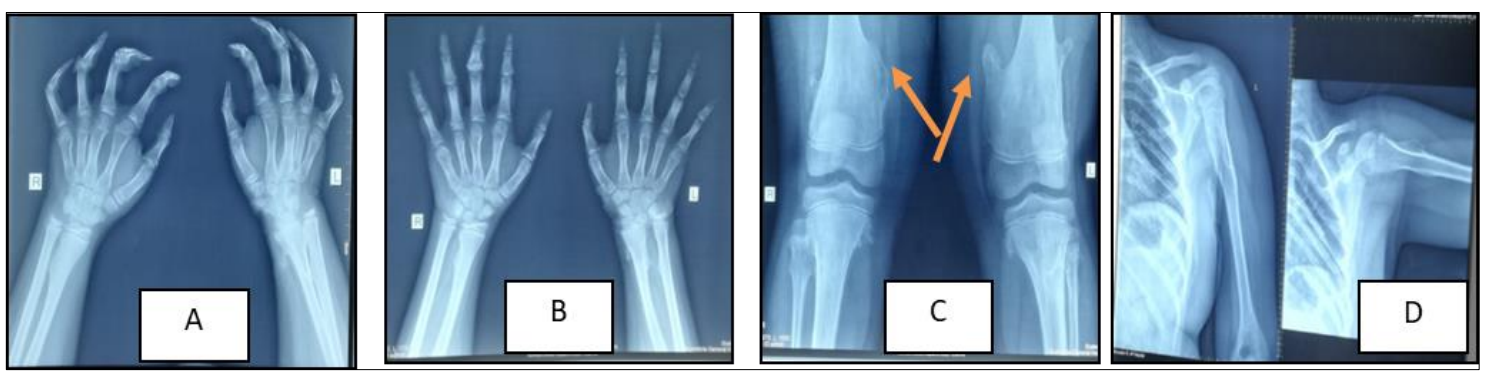

Figure 2 X-ray of both hands including both wrist joints (A,B), both knee joints (C) and left shoulder joint (D) showing bony overgrowth involving middle phalanx of right middle finger $(A, B)$, both femur $(C)$ and left humerus (D). These overgrowths are projecting from metaphysis, cortical continuity with the parent bone and pointing away from the joint (orange arrow)

\subsection{Radiographic examination}

Radiographic examination revealed multiple bony outgrowth extending from metaphysis of long bones involving the humerus (Figure 2D), both femur (Figure 2C) and middle phalanx of middle finger of right side (Figure 2A,B). Pedunculated variant is noted over the lateral aspect of both femur, directed away from the joint (Figure 2C). Cortex 
and medulla of the bony projections are continuous with that of the parent bones without any cortical breakage. Joint spaces are maintained. soft tissue appear normal.

\subsection{Laboratory investigations}

Laboratory studies showed : Complete blood count - hemoglobin \% $10.6 \mathrm{gm} / \mathrm{dl}$, ESR $67 \mathrm{~mm}$ in $1^{\text {st }} \mathrm{hr}$, white blood cell count 7,340/mm3 (neutrophil 37.2\%, lymphocyte 54\%), platelet count 1,54,000/mm3; CRP <6 ng/L, ferritin - 172 $\mathrm{ng} / \mathrm{ml}$, D- Dimer $0.64 \mathrm{mg} / \mathrm{L}$, RT-PCR positive for SARS-CoV-2. Biochemical parameters showed serum calcium $8.4 \mathrm{mg} / \mathrm{dl}$, serum P04 $5.4 \mathrm{mg} / \mathrm{dl}$, serum ALP $147 \mathrm{U} / \mathrm{L}$, serum PTH $51.6 \mathrm{pg} / \mathrm{ml}$, serum Vit D (25 - hydroxyl cholecalciferol) 24.21 $\mathrm{nmol} / \mathrm{L}$ (Insufficiency), serum creatinine $0.5 \mathrm{mg} / \mathrm{dl}$, serum electrolyte - within normal limit, SGPT 77 U/L.

Diagnosis of Hereditary Multiple Exostosis was made according to clinical and radiographic findings. The boy was managed with supportive and symptomatic treatment- paracetamol, calcium and vitamin-D supplementation. His covid status was improved. Then he was discharged with advice for home isolation and weight reduction. vitamin $\mathrm{D}$ supplementation was continued. A mandatory orthopedic consultation was planned for the patient at the age of $18 y e a r s$ along with a periodic follow up schedule (6-12 months).

\section{Discussion}

HME is a rare bone disease in pediatric population characterized by presence of multiple benign bone tumors called exostoses or osteochondromas [1,2]. Contrastingly, the solitary lesion is common and the growth pattern of it is comparable to that of HME [4].

It is a genetic disease, transmitted as an autosomal dominant pattern, with an estimated prevalence of 1 in 50,000 live birth. As most cases are asymptomatic, it seems that the value may be underestimated [1,3]. 50\% individuals with multiple osteochondromas are diagnosed before 3,5 years of age. The condition is diagnosed before the end of 1 st decade in more than $80 \%$ cases [7]. Diagnosis is made first at the age of 3 years in our case incidentally following trauma . A higher prevalence in male were found in previous studies, while recent studies found no sexual predilection [7,10,11]. A significant correlation was found between male sex and severity of clinical presentation that can be explained by hormonal differences between gender $[3,12]$. This is like the case of ours.

The pathogenesis is linked to loss of function mutation of 2 genes - Exostosin 1 (EXT1) \& Exostosin 2 (EXT2). This results in lower production of heparin sulfate. Disturbance in the heparan sulfate chain elongation is the cause of formation of exostoses. Severity of the disease is inversely correlated to heparan sulfate level $[13,14]$.

Mostly patients remain asymptomatic $(6,7)$. The primary clinical feature is multiple hard palpable mass arising from the ends of long bone (4). Involvement of carpal and tarsal bones are rare, never in the facial bones, because they develop by intramembranous ossification. Children mostly present with an average of 6 exoxtoses. The usual site of exostoses includes the distal femur (90\%), proximal tibia (84\%), fibula (76\%) and humerus (72\%). They are often discovered primarily over the ribs and the proximal tibia where they can be clearly visible and palpable. $[1,3]$.

Exostoses can cause a variety of health problems including skeletal deformities, growth retardation, painful joint motion, joint subluxation, neurovascular compression etc. $[15,16]$. skeletal deformities include leg length discrepancy, angular deformities around the knee, ankle and forearm, short stature, scoliosis [4]. The typical deformity of upper limb is ulnar shortening that may lead to subluxation and dislocation of radial head, highly curved radius. The condition is described as madelung - type of deformity. The pattern of involvement of lower limb is coxa valga deformity of hip joint and genu valgum deformity of knee joint. The rate of development of early knee arthritis and patellar subluxation is also high [17]. Genu valgum deformity may be noted nearly one-third of patients like ours (Figure 1D). [18].

A clinical classification system (Table 1) has been developed recently depending upon the number of involved bony segments and the presence of skeletal deformities and/or functional limitations. According to this classification system, HME is divided into three main classes/groups [3,19]. Our case falls into class IIIB as he had both deformities and functional limitations with involvement of $>5$ sites [2].

Earlier diagnosis is based upon genetic screening. Conventional radiograph is a good tool for accurately detecting the location, size and associated deformities [5]. They will visualize as a cartilage - capped bony overgrowth, continuing with the parent bone $[15,16]$. CT scan and MRI can be considered for better visualization of anatomically challenging regions (thorax, spine, pelvis). 
Table 1 Clinical classification of HME

\begin{tabular}{|l|l|}
\hline No deformities and no functional limitations & A $<5$ sites with osteochondromas \\
\cline { 2 - 2 } & B $>5$ sites with osteochondromas \\
\hline Deformities and no functional limitations & A $<5$ sites with deformities \\
\cline { 2 - 2 } & B $>5$ sites with deformities \\
\hline Deformities and functional limitations & A Functional limitation of 1 site \\
\cline { 2 - 2 } & B Functional limitation of $>1$ site \\
\hline
\end{tabular}

MRI provide better resolution for precise study of cartilage cap and soft tissues surrounding the lesion. [5]. The diagnosis can be made by clinical and radiological findings in absence of genetic proof like this case.

There is no specific treatment. Treatment is only conservative, while no or mild clinical problem exist [1]. Surgical resection of exostoses from the bone base along with cartilage cap is the choice of treatment when there is profound symptoms and a strong suspicion of malignancy [5,20]. Sometimes more complex procedure may be instituted to correct deformities, malalignment and leg length discrepancy in children [3]. Only mild analgesic, like paracetamol was instituted for our case as symptoms were mild initially [2].

Spontaneous regression of the exostoses has been reported in a single case during childhood and adolescence [21,22]. During childhood, they will increase in size and will stop enlarging at the end of normal growth period. Malignant transformation is a rare, but important complication, which may occur in $0.5-5 \%$ cases after the end of growth period $[1,3]$. The prognosis is good for this kind of malignancy as metastasis is rare. 5 year survival rate is $90 \%$ [2]. So, careful monitoring is necessary for our case until closure of the growth plate.

Considerable effects have been noted on different aspects of rare and complex diseases during covid-19 pandemic. It has been proven that pre-existing diseases and several co-morbidities are associated with worst clinical outcome in Covid-19 disease. The need to provide high quality care has become a challenge for these patients as well as their caregivers during this global health emergency [23].

\section{Conclusion}

HME is a rare and chronic disease. Covid-19 may affect at any age or with any disease. Reports about SARS-CoV-2 infection in children with HME are actually lacking. Patients with this sort of rare bone disease require continuous care and multidisciplinary approach for management. The pediatrician, the geneticist and orthopedic surgeon should take part simultaneously in diagnostic, therapeutic and research purpose. This case highlighted the importance of monitoring of these patients for detection of complications, appropriate intervention and preventing co-morbidities earlier.

\section{Compliance with ethical standards}

\section{Acknowledgments}

We would like to convey our heartfelt appreciation for the participant assistance. We are also thankful to Farzana Yeasmin Mukta, Register, department of cardiology, Kurmitola general hospital, Dhaka, Bangladesh for her supports and encouragements throughout the work.

\section{Disclosure of conflict of interest}

We announce that none of the authors have a financial or scientific conflict of interest.

\section{Statement of informed consent}

Informed consent was obtained from the individual participant included in the study. 


\section{References}

[1] Nassar K, Janani S. Multiple exostosis Disease. Case. Rep. Int.J. Clin. Rheumatol. 2021.

[2] Pacifici M. Hereditary multiple exostosis: are there new plausible treatment strategies? Expert. Opin. Orphan. Drugs. 2018; 6(6): 385 - 391.

[3] Beltrami G, Ristori G, Scoccianti G, Tamburini A, Capanna R. Heriditary multiple exostoses: a review of clinical appearance and metabolic pattern. Clin Cases Miner Bone Metab. 2016; 13 (2): 110-118.

[4] EL-Sobky TA, Samir S, Atiyya AN et al. Current paediatricorthopaedic practice in hereditary multiple osteochondromas of the forearm: a systematic review. SICOT-J. 2018; 4: 10.

[5] D’Arienzo A, Andreani L, Sacchetti F et al. Hereditary Multiple Exostoses: Current Insights. Orthop. Res. Rev. 2019; 11: 199-211.

[6] Wells M, Birchard Z. A 40-Year-Old Male Presenting with Hereditary Multiple Exostosis: Management and Considerations. Case. Rep. Orthop. 2019.

[7] Porter DE, Lonie L, Fraser $\mathrm{M}$ et al. Severity of disease and risk of malignant change in hereditary multiple exostoses. J. Bone. Joint. Surg. Br. 2004; 86(7): 1041-1046.

[8] Zuntini M, Pedrini E, Parra A, et al. Genetic models of osteochondroma onset and neoplastic progression: evidence for mechanisms alternative to EXT genes inactivation. Oncogene. 2010; 29 (26): 3827-3834.

[9] Rozeman LB, de Bruijn IH, Bacchini P, et al. Dedifferentiated peripheral chondrosarcomas: regulation of EXTdownstream molecules and differentiation related genes. Mod Path. 2009; 22: 1489-1498.

[10] Shapiro F, Simon S, Glimcher HJ. Hereditary multiple exostoses. Anthropometric, roentgenographic, and clinical aspects. J Bone Joint Surg Am. 1979; 61A: 815e24.

[11] Wicklund CL, Pauli RM, Johnston D, Hecht JT. Natural history study of hereditary multiple exostoses. Am J Med Genet. 1995; 55: $43 \mathrm{e} 6$.

[12] Pedrini E, Jennes I, Tremosini M, et al. Genotype-phenotype correlation study in 529 patients with multiple hereditary exostoses: identification of "protective" and "risk" factors. J Bone Joint Surg Am. 2011; 2011(93): $2294 \mathrm{e} 302$.

[13] Stickens D, Clines G, Burbee D, et al. The EXT2 multiple hereditary exostoses gene defines a family of putative tumor suppressor genes. Nat Genet. 1996; 14: 25-32.

[14] Zak BM, Schuksz M, Koyama E, et al. Compound heterozygous loss of Ext1 and Ext2 is sufficient for formation of multiple exostoses in mouse ribs and long bones. Bone. 2011; 48(5): 979-987.

[15] Guo XL, Deng Y, Liu HG. Clinical characteristics of hereditary multiple exostoses: a retrospective study of mainland chinese cases in recent 23 years. J Huazhong Univ Sci Technolog Med Sci. 2014; 34: 42-50.

[16] Jones KB. Glycobiology and the growth plate: current concepts in multiple hereditary exostoses. J Pediatr Orthop. 2011; 31: 577-586.

[17] Maurizio Pacifici. Hereditary Multiple Exostoses: New Insights into Pathogenesis, Clinical Complications, and Potential Treatments. Curr.Osteoporos. Rep. 2017; 15(3): 142- 152.

[18] Clement ND, Porter DE. Can deformity of the knee and longitudinal growth of the leg be predicted in patients with hereditary multiple exostoses? A cross-sectional study. The Knee. 2014; 21: 299-303.

[19] Mordenti M, Ferrari E, Pedrini E, et al. Validation of a new multiple osteochondromas classification through Switching Neural Networks. Am J Med Genet. 2013; 161A: 556-560.

[20] Jager M, Westhoff B, Portier S, et al. Clinical outcome and genotype in patients with hereditary multiple exostoses. Clinical outcome and genotype in patients with hereditary multiple exostoses. J Orthop Res. 2007; 25: 1541-51.

[21] Passanise AM, Mehlman CT, Wall EJ, et al. Radiographic evidence of regression of a solitary osteochondroma: a report of 4 cases and a literature review. J Pediatr Orthop. 2011; 31: 312-6.

[22] Gigi R, Kurian BT, Cole A, et al. Late presentation of spinal cord compression in hereditary multiple exostosis: case reports and review of the literature. J. Child .Orthop. 2019; 13: 463-470.

[23] Brizola E, Adami G, Baroncelli GI, et al. Providing high quality care remotely to patients with rare bone diseases during COVID-19 pandemic. Orphanet J Rare Dis. 2020; 15(1): 228. 